\title{
COMMENTARY
}

\section{Reducing the global burden of sepsis}

\author{
Sagar Dugani MD PhD, Jeremy Veillard PhD, Niranjan Kissoon MBBS
}

Cite as: CMAJ 2017 January 9;189:E2-3. doi: 10.1503/cmaj.160798

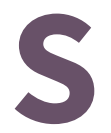

epsis is a burdensome condition worldwide in terms of morbidity, mortality and financial cost to health systems. A recent global task force report that was endorsed by 31 clinical societies defined sepsis as "life-threatening organ dysfunction caused by a dysregulated host response to infection." 1 This definition reflects impairment in organ function, but does not identify the cause of the impairment; sepsis may result from any systemic infection, not only bacterial infections. We advocate for inclusion of sepsis as a separate cause of morbidity and mortality in frameworks that focus on the global burden of disease.

Although different organisms have varying virulence, overall sepsis is associated with substantial hospital mortality of $25 \%$ $30 \%^{2}$ globally, which increases to $40 \%-50 \%$ in patients with complications ${ }^{2}$ and in lower-income countries. ${ }^{3}$ An estimated 30 million cases of sepsis each year result in more than 8 million deaths. This number is likely an underestimate, however, because data on incidence and outcomes are often poorly captured in low- and middleincome countries where access to care is difficult. ${ }^{4}$ In the United States alone, spending on sepsis was estimated at US $\$ 20$ billion (5.2\% of hospital costs) in $2011 .{ }^{5}$ Despite its burden, sepsis is not well recognized as a leading cause of death in its own right.

The collection of data on diagnosis, management and outcomes specifically related to sepsis needs to be improved in line with its global burden. Furthermore, better sharing of data and experiences across health systems is required to make headway on reducing rates.

Sepsis may result from, and is the final common pathway for death and disability from, various infections including malaria, respiratory tract infections, urinary tract infections and gastrointestinal infections. Consequently, various traditional studies focus on morbidity and mortality outcomes for single disease processes, and then link to preventive measures and treatment strategies for specific diseases. For example, the Global Burden of Disease study recently characterized the leading causes of global morbidity and mortality, ${ }^{6}$ describing sepsis from the lens of neonatal and maternal health. ${ }^{6}$ Although it is important to identify the burden of individual diseases (for example, tuberculosis or pneumonia), we fail to examine the burden fully if we do not consider sepsis as a separate contributor to morbidity and mortality. What's more, without adequate study of the particular risk factors for and outcomes following sepsis, we cannot develop strategies for its optimal treatment.

When health care providers encounter a critically ill patient, identifying the cause of sepsis is less important than the screening

\section{KEY POINTS}

- Sepsis is a leading killer worldwide, accounting for about 8 million deaths each year.

- Mortality for severe sepsis is between $15 \%$ and $30 \%$ in highincome countries; it is $50 \%$ or higher in low-income countries.

- Health care providers must improve upon diagnosis, context-based research and treatment options and follow-up for mortality and morbidity after a patient's discharge from hospital.

- Broader engagement with all segments of society, including multilateral organizations and donor and charitable organizations engaged in global health, is needed for advocacy to combat sepsis locally and globally.

and initial management of the common deranged physiology that characterizes sepsis regardless of its cause. This approach is even more relevant for health care workers in low- and middle-income countries, who have limited resources with which to manage patient care. For this reason, the new definition of sepsis requires the presence of just two simple clinical features - increased respiratory rate, altered mental status or reduced blood pressure - to screen for the presence of sepsis. ${ }^{1}$ If screening is positive, additional simple bedside tests and blood tests can be used to make a diagnosis. The Surviving Sepsis Campaign ${ }^{7}$ has outlined intervention bundles that should be given within three to six hours of a patient presenting with sepsis. Notably, adequate sepsis management can begin without identifying the underlying cause or microorganism. Despite the various causes, patients with sepsis and septic shock exhibit profound cardiovascular dysfunction, and the approach to their initial care is similar. For example, initial care includes early administration of broad-spectrum antibiotics, infusion of intravenous fluids to maintain hemodynamics and reduce further organ dysfunction, and achieving source control when possible. A research agenda that identifies sepsis as a major contributor to mortality in its own right would help to shift focus away from determining cause and toward rapid resuscitation with interventions that could save lives.

As protocols for sepsis become well defined, categorizing sepsis as a disease may unify and organize health facility frameworks required to manage sepsis. The need for structural facilities (properly equipped emergency departments and critical care units), adequately trained health care providers, intravenous fluids and 
necessary antibiotics, and the financial resources to support these, becomes clear. A focus on clearly identifying a patient as having sepsis will help to ensure that health care providers recognize that the patient is critically ill so as to begin rapid mobilization of lifesaving therapies. In addition, developing a framework around sepsis allows hospitals to develop care plans and to monitor, evaluate and improve their timely delivery.

In an effort to advocate for greater awareness of sepsis and the importance of its individual categorization as a condition of global burden, the Global Sepsis Alliance ${ }^{8}$ (GSA) is raising awareness through its World Sepsis Day campaign and World Sepsis Declaration. The alliance is calling for the development of national and international registries, and for broad engagement with policymakers and governments to identify opportunities to improve data collection, care processes and outcomes for patients with sepsis. The United Kingdom has developed a series of measures to improve outcomes from sepsis; in Germany, a nationwide action plan has been formulated after a vigorous campaign by clinicians and policymakers. ${ }^{8}$ The Global Sepsis Alliance is engaged in efforts to obtain a United Nations resolution that would lead to the recognition of sepsis as a major public health threat and lead to increased attention from governments and multilateral organizations.

Sepsis should be identified as a separate contributor to disability and death. Progress has been made in recent years to describe sepsis and septic shock, and to outline the initial man- agement of sepsis. ${ }^{1}$ International organizations have developed management algorithms, which could help standardize treatment and monitor progress toward delivery of timely care. However, shifting the spotlight to sepsis as a distinct entity may set the stage for a robust response to this important global contributor to the burden of death and illness.

\section{References}

1. Singer M, Deutschman CS, Seymour CW, et al. The Third International Consensus Definitions for Sepsis and Septic Shock (Sepsis-3). JAMA 2016;315:801-10.

2. Cohen J, Vincent J-L, Adhikari NKJ, et al. Sepsis: a roadmap for future research. Lancet Infect Dis 2015;15:581-614.

3. Kwizera A, Dünser M, Nakibuuka J. National intensive care unit bed capacity and ICU patient characteristics in a low income country. BMC Res Notes 2012; 5:475.

4. Fleischmann C, Scherag A, Adhikari NKJ, et al. Assessment of global incidence and mortality of hospital-treated sepsis. Current estimates and limitations. Am J Respir Crit Care Med 2016;193:259-72.

5. Torio CM, Andrews RM. National inpatient hospital costs: the most expensive conditions by payer, 2011: statistical brief \#160. Rockville (MD): Agency for Healthcare Research and Quality; 2013.

6. Murray CJL, Barber RM, Foreman KJ, et al. Global, regional, and national disabilityadjusted life years (DALYs) for 306 diseases and injuries and healthy life expectancy (HALE) for 188 countries, 1990-2013: quantifying the epidemiological transition. Lancet 2015;386:2145-91.

7. Surviving sepsis campaign. Mt Prospect (IL): Society of Critical Care Medicine. Available: www.survivingsepsis.org/Pages/default.aspx (accessed 2016 June 2).

8. World sepsis day: a call to action. Jena (Germany): Global Sepsis Alliance for the World Sepsis Day. Available: http://world-sepsis-day.org/?MET=HOME \&VLANGUAGE=EN (accessed 2016 Oct. 25).

\section{Competing interests: None declared.}

This article has been peer reviewed.

Disclaimer: Sagar Dugani and Niranjan Kissoon are members of the CMAJ Editorial Advisory Board and were not involved in the editorial decision-making process for this article.
Affiliations: Division of Internal Medicine (Dugani), St Michael's Hospital, University of Toronto, Toronto, Ont.; Institute of Health Policy, Management and Evaluation (Veillard), Dalla Lana School of Public Health, University of Toronto, Toronto, Ont.; Health, Nutrition and Population Global Practice (Veillard), The World Bank Group, Washington DC; University of British Columbia and British Columbia Children's Hospital (Kissoon), Vancouver, BC
Contributors: Niranjan Kissoon contributed to the concept and ideas for the article. Sagar Dugani drafted the manuscript. All of the authors contributed to critical revisions of the manuscript for important intellectual content, approved the final version to be published and agreed to act as guarantors of the results.

Correspondence to: Niranjan Kissoon, nkissoon@cw.bc.ca 